\title{
Phytoprotection
}

\section{Variabilité du virus de la striure du maïs (MSV) en zone soudano-sahélienne}

\section{G. Konaté et O. Traoré}

Volume 75, numéro 2, 1994

URI : https://id.erudit.org/iderudit/706055ar

DOI : https://doi.org/10.7202/706055ar

Aller au sommaire du numéro

Éditeur(s)

Société de protection des plantes du Québec (SPPQ)l

ISSN

0031-9511 (imprimé)

1710-1603 (numérique)

Découvrir la revue

Citer cet article

Konaté, G. \& Traoré, O. (1994). Variabilité du virus de la striure du maïs (MSV) en zone soudano-sahélienne. Phytoprotection, 75(2), 91-99.

https://doi.org/10.7202/706055ar
Résumé de l'article

Une étude de la variabilité du virus de la striure du maïs (MSV) a été réalisée au Burkina Faso, situé dans la zone soudano-sahélienne. De 1990 à 1992, 1240 échantillons ont été prélevés sur 36 hôtes naturellement infectés par le virus. Ils ont été analysés à l'aide du test ELISA, en utilisant cinq anticorps monoclonaux préparés contre le virus et par transmission du virus à quelques hôtes naturels. Trois variants sérologiques du virus, désignés par SK, VD114 et VD180, ont été caractérisés. D'autre part, il s'est avéré qu'il existait deux formes symptomatiques du variant SK soit $\mathrm{SK}_{\mathrm{F}}$ induisant des symptômes prononcés et $\mathrm{SK}_{\mathrm{f}}$, des symptômes faibles. Chez le maïs (Zea mays), en infection mixte, le variant SK masquait les deux autres variants du MSV. L'apparente uniformité antigénique des isolats MSV du maïs observée par plusieurs auteurs a été attribuée à ce phénomène de masquage. 


\title{
Variabilité du virus de la striure de maïs (MSV) en zone soudano-sahélienne
}

\author{
Gnissa Konaté ${ }^{1}$ et Oumar Traoré ${ }^{1}$
}

Reçu 1993-04-07; accepté 1993-12-24

Une étude de la variabilité du virus de la striure du maïs (MSV) a été réalisée au Burkina Faso, situé dans la zone soudano-sahélienne. De 1990 à 1992, 1240 échantillons ont été prélevés sur 36 hôtes naturellement infectés par le virus. Ils ont été analysés à l'aide du test ELISA, en utilisant cinq anticorps monoclonaux préparés contre le virus et par transmission du virus à quelques hôtes naturels. Trois variants sérologiques du virus, désignés par SK, VD114 et VD180, ont été caractérisés. D'autre part, il s'est avéré qu'il existait deux formes symptomatiques du variant SK soit $\mathrm{SK}_{\mathrm{F}}$ induisant des symptômes prononcés et $\mathrm{SK}_{\mathrm{f}}$, des symptômes faibles. Chez le maïs (Zea mays), en infection mixte, le variant SK masquait les deux autres variants du MSV. L'apparente uniformité antigénique des isolats MSV du maïs observée par plusieurs auteurs a été attribuée à ce phénomène de masquage.

Konaté, G., and O. Traoré. 1994. Variability of the maize streak virus (MSV) in the Sudano-Sahelian region. PHYTOPROTECTION 75: 91-99.

A study of the variability of the maize streak virus (MSV) was conducted in Burkina Faso, which is part of the Sudano-Sahelian region. From 1990 to 1992,1240 samples were collected on 36 hosts naturally infected by the virus. These samples were analysed with ELISA, using five monoclonal antibodies developed against the MSV and by transmission of the virus to some natural hosts. Three serological variants of the virus, designated as SK, VD114, and VD180 were characterized. The SK variant was further divided into two forms, namely $\mathrm{SK}_{\mathrm{F}}$ and $\mathrm{SK}_{\mathrm{f}}$, which induced severe and mild symptoms, respectively. In maize (Zea mays), with multiple infection, the SK variant masked the other two MSV variants. The apparent antigenic uniformity of the MSV isolates from maize observed by many authors has been linked with this masking effect.

\section{INTRODUCTION}

La striure du maïs est la maladie virale de cette plante (Zea mays L.) la plus anciennement connue en Afrique. Elle a été décrite dès le début du $X X^{\mathrm{e}}$ siècle par Fuller (1901) au Natal, en Afrique du Sud, sous le nom de mealie variegation. Elle est considérée aujourd'hui comme la principale maladie virale de cette céréale en Afrique et dans les Mascareignes.

L'agent pathogène responsable de la maladie est un geminivirus (Francki et al. 1985; Harrison 1985; Stanley 1985) dont le génome est formé d'une seule molécule d'ADN circulaire de 2,7 kb (Mullineaux et al. 1984), et la capside, d'une protéine de $26 \mathrm{kD}$. II est transmis obligatoirement par plusieurs espèces

1. Institut d'Études et de Recherches Agricoles - INERA, 03, B.P. 7192, Ouagadougou 03, Burkina Faso 
de cicadelles appartenant toutes au genre Cicadulina Naude (Rose 1978; Webb 1987). Excepté pour le virus de la striure du Digitaria sanguinalis (L.) Scop. (DSV) (Dollet et al. 1986), le virus de la striure du maïs (MSV) n'a de relation sérologique avec aucun geminivirus connu (Harrison 1985; Roberts et al. 1984).

La variabilité du MSV a fait l'objet de nombreuses études. Les résultats obtenus indiquent qu'il existe plusieurs isolats infectant le maïs et des graminées sauvages. Ces isolats diffèrent principalement par leur virulence face au maïs et par leur gamme d'hôtes (Bock et al. 1974; Dekker et al. 1988; McClean 1947; Peterschmitt et al. 1991; Pinner et Markham 1990; Pinner et al. 1988; Ricaud et Félix 1978; Seth et al. 1972; Storey et McClean 1930). Du point de vue sérologique, tous les isolats connus sont reliés, mais des épitopes spécifiques à certains isolats ont été rapportés (Dekker et al. 1988; Peterschmitt et al. 1991; Pinner et Markham 1990; Pinner et al. 1988).

Contrairement aux isolats MSV infectant les graminées sauvages, il a été rapporté que les isolats MSV du maïs sont difficiles, voire impossibles à distinguer les uns des autres sur la base de leurs propriétés biologiques et sérologiques (Dekker et al. 1988; Peterschmitt et al. 1991; Pinner et al. 1988). L'explication de ce phénomène n'est pas connue. D'autre part, on sait peu de choses sur la prévalence des isolats du MSV ainsi que sur leurs interactions dans la nature. Par conséquent I'objectif des travaux rapportés ici était: 1) d'étudier la variabilité du MSV dans la zone soudano-sahélienne et d'expliquer pourquoi les isolats MSV du maïs sont difficiles à distinguer les uns des autres; et 2) de rechercher les interactions entre les isolats du MSV en condition d'infection naturelle.

\section{MATÉRIEL ET MÉTHODES}

\section{Source et transmission du MSV}

La collecte des échantillons de plantes portant les symptômes de la striure du maïs a été réalisée au Burkina Faso à partir de 36 hôtes naturels du virus (Konaté et Traoré 1992) durant les années 1990, 1991 et 1992. Dans toutes les expériences de transmission, le Cicadulina triangula Ruppel [Homoptera: Cicadellidae] a été utilisé comme vecteur. Deux techniques de transmission ont été utilisées: dans la prernière technique, dite ordinaire, 50 insectes sains privés de nourriture pendarit $3 \mathrm{~h}$ ont été déposés sur la source d'inoculum pendant $48 \mathrm{~h}$ pour acquisition du virus. Ils ont été ensuite transférées sur I'hôte à infecter pendant $48 \mathrm{~h}$. Les insectes ont alors été tués par un traitement de deltaméthrine ((S) $\alpha$-cyano3-phénoxybenzyl(1R,3R)-3(2,2-dibromovinyl)-2, 2-diméthylcyclopropanecarboxylate) à $12 \mathrm{~g} \mathrm{~m} . a . \mathrm{L}^{-1}$. Dans la deuxième technique dite spéciale, le temps $d^{\prime}$ acquisition du virus par le vecteur n'a été que de $5 \mathrm{~min}$. Les insectes ont été alors transférés individuellement.

\section{Variation antigénique du MSV}

Les échantillons collectés ont été d'abord analysés pour la présence du MSV à I'aide de la variante de l'essai immuno-enzymatique ELISA décrite par Voller et al. (1976) en utilisant des anticorps polyclonaux de lapin préparés contre un isolat maïs du MSV de l'île de la Réunion. Les échantillons ayant donné une réponse positive ont été ensuite analysés à l'aide de la variante du rnême test décrite par Van Regenmortel et Burckard (1980) en utilisant cinq anticorps monoclonaux préparés contre le même isolat. Ces anticorps monoclonaux ont été nommés $114 \times 1,114 \times 2$, $94 \times 2,180 \times 3$ et $180 \times 4$ (Peterschmitt et al. 1991). Les tampons utilisés pour le deuxième procédé ELISA ont été ceux décrits par Clark et Adams (1977). Dans toutes les expériences, les extraits de feuille saine ou malade ont été obtenus en broyant $1 \mathrm{~g}$ de feuille dans $10 \mathrm{~mL}$ de tampon phosphate salin-Tween. L'homogénat obtenu a été centrifugé à $10000 \mathrm{~g}$ pendant $10 \mathrm{~min}$ à $4^{\circ} \mathrm{C}$. Les plaques de microtitration (NUNC F96, A/S NUNC, Kamstrup, DK-4000 Rioskilde, Danemark), préalablement recouvertes avec des IgG polyclonaux de lapin à $1 \mu \mathrm{g} \mathrm{mL}^{-1}$ ont été saturées avec de l'ovalbumine de poule à $10 \mathrm{~g} \mathrm{~L}^{-1}$ pendant $30 \mathrm{~min}$. Après avoir capté le virus 
sur les plaques, les anticorps monoclonaux de souris ont été incubés aux dilutions de $10^{5}$ pour les anticorps $114 \times 1$, $114 \times 2$ et $94 \times 2$, et $10^{4}$ pour les anticorps $180 \times 3$ et $180 \times 4$ (Peterschmitt et al. 1991). L'anticorps de détection couplé à la phosphatase alcaline était constitué $d^{\prime} \lg G$ de chèvre anti-souris. Le substrat de l'enzyme, le para-nitrophénylphosphate à $1 \mathrm{mg} \mathrm{mL}^{-1}$, a été incubé pendant $1 \mathrm{~h}$ à $37^{\circ} \mathrm{C}$. La variation des épitopes du MSV a été évaluée en se basant sur l'intensité de l'absorbance due à chaque échantillon en fonction de I'anticorps monoclonal, mesurée avec un lecteur automatique de plaque (Uniskan II, Labsystems).

\section{Reconstitution de profils de réaction hybrides}

Des feuilles prélevées sur des plants de maïs infectés, les uns par SK et les autres par VD114 depuis 3 sem, ont été broyées séparément dans du tampon phosphate salin-Tween selon le même rapport (p:vol). Des portions de l'extrait de feuille infectée par SK ont été diluées 100 et 500 fois. L'extrait de feuille infectée par VD114 n'a pas été dilué. On a mélangé un même volume de l'extrait VD114 et de l'extrait SK aux différentes dilutions. Les mélanges et les extraits purs ont alors été analysés par ELISA en utilisant les cinq anticorps monoclonaux, tel que décrit précédemment.

\section{Épuisement sérologique}

Un extrait exhibant le profil de réaction hybride SK $\times$ VD114 a été incubé en présence de l'anticorps monoclonal $114 \times 1$, dilué 250 fois, pendant $2 \mathrm{~h}$. Le mélange a été ensuite déposé dans des alvéoles de plaque de microtitration (NUNC F96) préalablement recouvertes avec des Ig G de chèvre anti-souris pendant $2 \mathrm{~h}$, puis transféré dans de nouvelles alvéoles pendant le même temps. L'extrait ainsi traité a été analysé en ELISA à l'aide des cinq anticorps monoclonaux.

\section{RÉSULTATS}

\section{Variation antigénique du MSV}

Les anticorps polyclonaux de lapin antiMSV isolat maïs de l'île de la Réunion ont réagi positivement avec les 1240 échantillons de feuille prélevés sur 33 espèces de graminées sauvages et sur trois espèces de graminées cultivées. L'analyse des mêmes échantillons à I'aide des cinq anticorps monoclonaux a mis en évidence trois profils de réaction différents (fig. 1). Un $1^{\text {er }}$ profil est caractérisé par des réactions fortes et sensiblement de même niveau avec les cinq anticorps. Dans un $2^{\mathrm{e}}$ profil, l'intensité de la réaction avec les anticorps $114 \times 1$ et $114 \times 2$ a été nulle alors que celle de la réaction avec les trois autres a été forte et sensiblement de même niveau. Quant au $3^{e}$ profil, il est distingué des deux précédents par une intensité de réaction nulle avec les anticorps $180 \times 3$ et $180 \times 4$, mais forte avec les trois autres. Ces trois profils de réaction ont été attribués à trois variants sérologiques du MSV. II a été convenu de les nommer respectivement SK (pour Station de Kamboinsé), VD114 et VD180 (pour variants sérologiques défectueux pour la reconnaissance par les anticorps 114 et 180 , respectivement). Pour un échantillon donné, les anticorps monoclonaux $114 \times 1$ et $114 \times 2$ ont eu le même comportement. II en a été de même pour $180 \times 3$ et $180 \times 4$. L'intensité de la réaction avec $94 \times 2$ est restée constante, peu importe l'échantillon analysé.

\section{Virulence des variants sérologiques}

Le tableau 1 montre les résultats de l'étude de la virulence des variants SK, VD114 et VD180 vis-à-vis de la lignée sensible de maïs M $162 \mathrm{~W}$. Pour le variant SK, des isolats ont manifesté deux niveaux de virulence, I'un sévère et l'autre faible. II a été convenu d'attribuer ces deux niveaux de virulence à deux sous-variants symptomatiques $\mathrm{SK}_{\mathrm{F}}$ et $\mathrm{SK}_{\mathrm{f}}$, respectivement. Les variants VD114 et VD180, bien que sérologiquement distincts, ont manifesté le même niveau de virulence atténué. Le sousvariant $\mathrm{SK}_{\mathrm{f}}$ a été rencontré préférentiellement sur les hôtes appartenant au genre Digitaria et occasionnellement sur le maïs. Dans la quasi-totalité des champs inspectés, les plants infectés par le MSV portaient les symptômes forts de $\mathrm{SK}_{\mathrm{F}}$.

\section{Hôtes spécifiques du VD114}

Les hôtes suivants: Brachiaria deflexa (Schumach.) Hubb. ex. Robyns, Oryza 


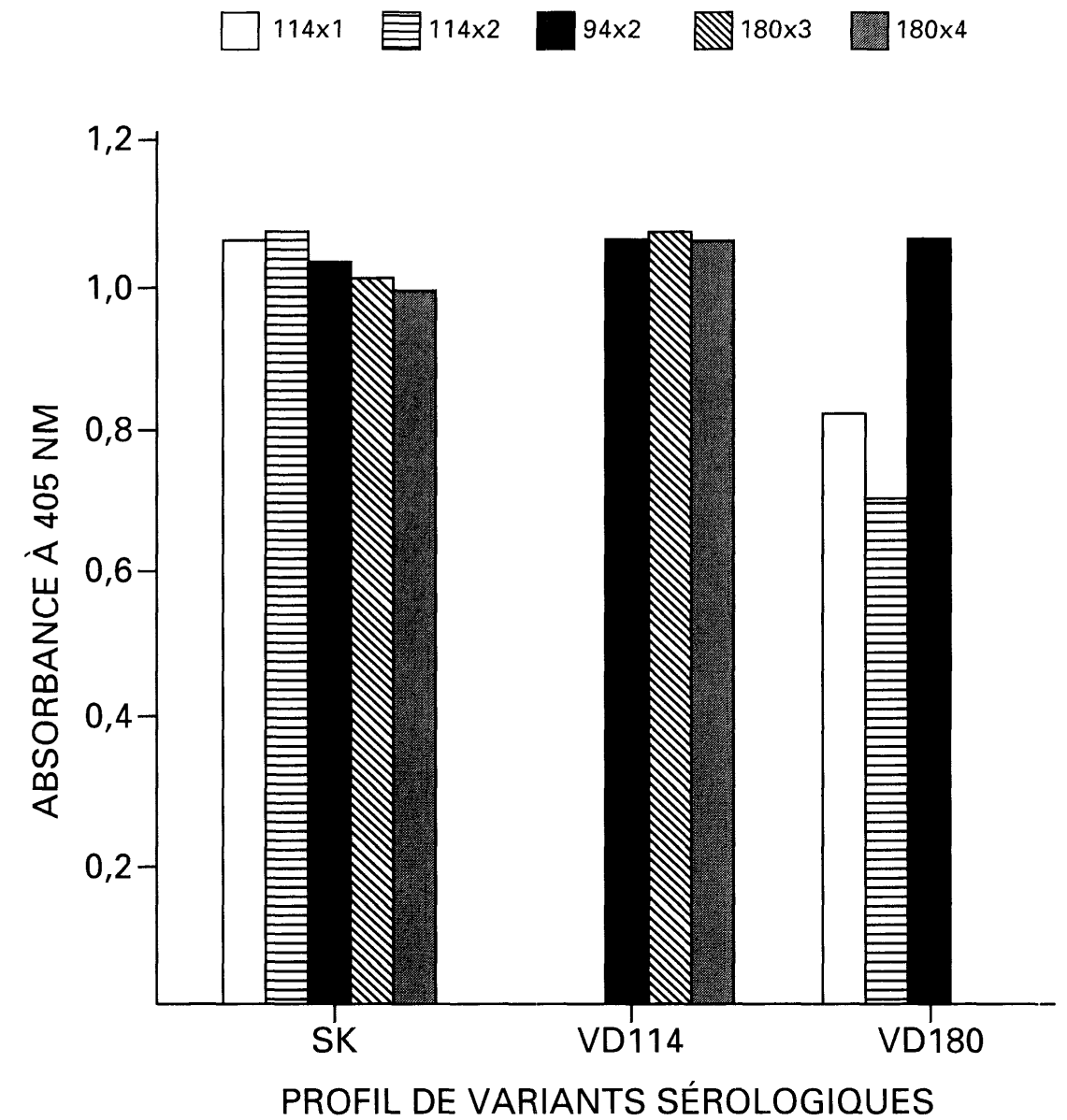

Figure 1. Types de profils de réaction ELISA, incluant cinq anticorps monoclonaux anti-virus de la striure du maïs (MSV). Ces profils ont été obtenus avec des échantillons proveriant de 36 hôtes naturellement infectés par le virus.

sativa L., Pennisetum glaucum (L.) R. Br., Rottboellia exaltata L.f. et Setaria pallide-fusca (Schumach.) Stapf et Hubb. ont été trouvés infectés seulement par le variant VD114. II n'a pas été possible de transmettre les variants SK et VD180 à ces cinq hôtes (tableau 2) qui peuvent être considérés, de ce fait, comme des hôtes spécifiques du variant VD114.

\section{Interactions entre les variants du MSV}

Plusieurs échantillons prélevés sur les hôtes suivants: Eragrostis aspera (Jacq.) Nees, Eleusine indica (L.) Gaertn., Digitaria horizontalis Willd., Digitaria sp. et Setaria barbata Kunth, ont réagi moyennement soit avec $114 \times 1$ et $114 \times 2$ d'une part, soit avec $180 \times 3$ et $180 \times 4$ d'autre part, mais fortement avec les autres anticorps monoclonaux. L'utilisation de la technique de transmission du virus après une acquisition de 5 min a permis de mettre en évidence la présence simultanée dans ces échantillons, soit de SK $\mathrm{F}_{\mathrm{F}}$ et VD114, soit de $S_{\mathrm{F}}$ et VD180. Par ailleurs, il a été possible de reconstituer in vitro de tels profils de réaction en mélangeant, à des proportions précises, $S_{\mathrm{F}}$ et VD114 d'une part, $S K_{F}$ et VD180 d'autre part (fig. 2). De même, la technique d'épuisement sérologique a permis de rétablir 
Tableau 1. Virulence de trois variants sérologiques du MSV envers de la lignée de maïs M 162 W

\begin{tabular}{|c|c|c|}
\hline Variant & Symptômes & $\begin{array}{c}\text { Délai d'apparition des } \\
\text { symptômes } \\
\text { (j) }\end{array}$ \\
\hline \multicolumn{3}{|l|}{ SK } \\
\hline $\begin{array}{l}\text { sous-variant } \\
\mathrm{SK}_{\mathrm{F}}\end{array}$ & $\begin{array}{l}\text { Chlorose générale du limbe } \\
\text { laissant seulement des îlots ou } \\
\text { de fines lignes vertes et dis- } \\
\text { continues. } \\
\text { Symptômes permanents. }\end{array}$ & $5-6$ \\
\hline $\begin{array}{l}\text { sous-variant } \\
\text { SK }_{f}\end{array}$ & $\begin{array}{l}\text { Striure très fine avec stries for- } \\
\text { mées de petits tirets ou lignes } \\
\text { dépassant rarement } 1 \mathrm{~cm} \text { de } \\
\text { longueur. } \\
\text { Symptômes éphémères. }\end{array}$ & $5-6$ \\
\hline VD114 & $\begin{array}{l}\text { Stries fines translucides de } \\
\text { densité moyenne. } \\
\text { Symptômes permanents. }\end{array}$ & $10-12$ \\
\hline VD180 & $\begin{array}{l}\text { Stries fines translucides de } \\
\text { densité moyenne. } \\
\text { Symptômes permanents. }\end{array}$ & $10-12$ \\
\hline
\end{tabular}

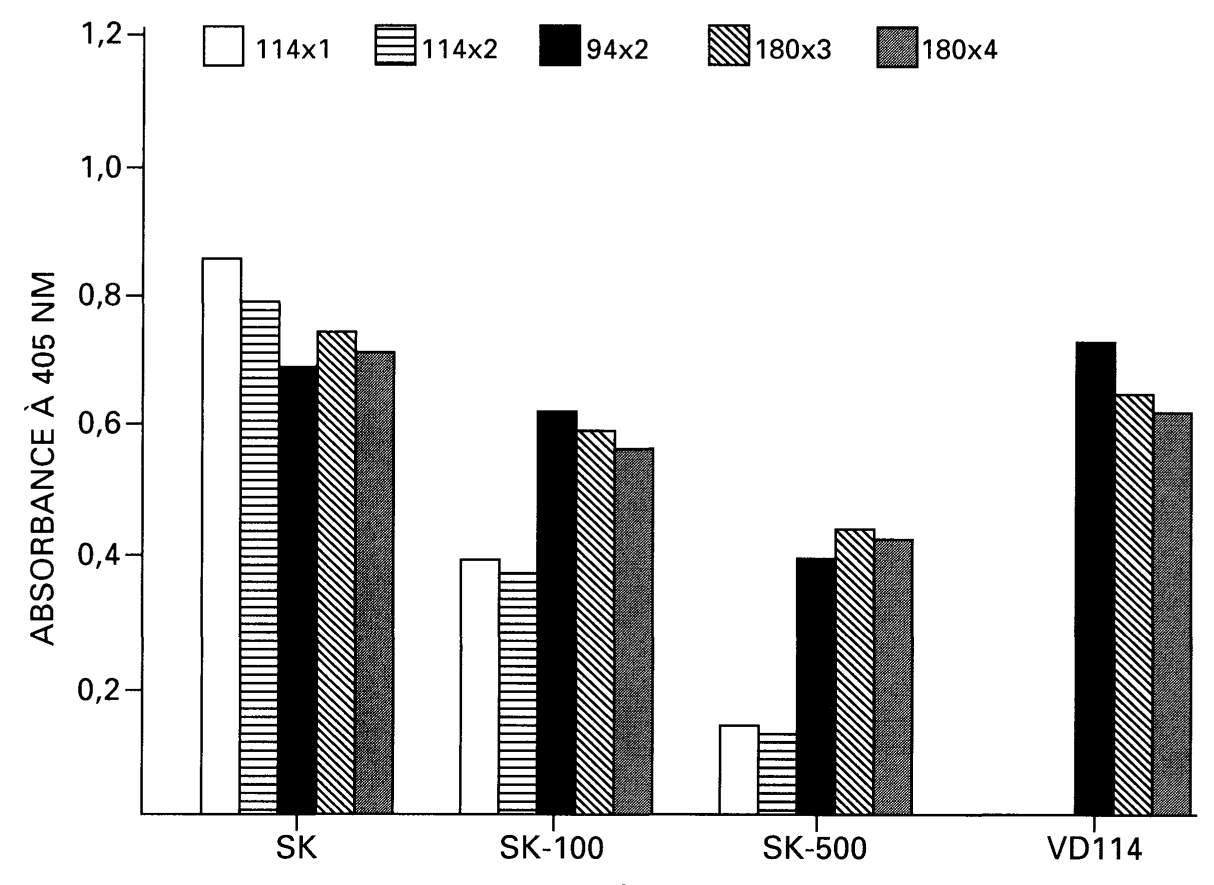

PROFIL DE VARIANTS SÉROLOGIQUES

Figure 2. Reconstitution, in vitro, du profil de réaction hybride SK x VD114. Profil SK: extrait SK non dilué; profils SK-100 et SK-500: SK dilué 100 et 500 fois respectivement; profil D: extrait VD114 non dilué. 
le profil VD114 à partir du profil hybride SK x VD114 (fig. 3). Bien que le maïs ait été trouvé infecté en condition naturelle par les trois variants sérologiques du MSV, aucun des 332 échantillons de maïs analysés n'a montré un tel profil de réaction. Cependant, l'utilisation d'un hôte spécifique du VD114, en I'occurrence $B$. deflexa, a montré que sur 112 échantillons de maïs examinés et exhibant le profil de réaction SK, 46 contenaient simultanément les variants SK et VD114. Par ailleurs, I'inoculation contrôlée simultanée ou décalée de $S_{K_{F}}$ et VD114 ou SK et VD180 au maïs a toujours abouti à des plants infectés portant sur les symptômes prononcés de $\mathrm{SK}_{\mathrm{F}}$ et exhibant le profil de réaction $S K$.

\section{DISCUSSION}

L'anticorps monoclonal $94 \times 2$ a réagi de façon constante avec tous les échantillons analysés. Cela signifie que l'épitope reconnu est peu variable. Les réactions identiques des anticorps $114 \times 1$ et $114 \times 2$ pour un même échantillon quel qu'il soit suggère qu'ils reconnaissent le même épitope. II en est de même pour les anticorps $180 \times 3$ et $180 \times 4$. Ceci confirme les observations de Peterschmitt et al. (1991). Aucun de nos échantillons $n$ 'a réagi faiblement et simultanément avec les quatre anticorps $114 \times 1$, $114 \times 2,180 \times 3$ et $180 \times 4$, contrairement aux résultats obtenus par Peterschmitt et al. (1991) avec un isolat canne à sucre (Saccharum officinarum L.) de l'île de la Réunion et avec le DSV.

Une partie des profils de réaction sérologique dans lesquels tous les anticorps ont réagi, résultent d'une complémentation sérologique partielle du variant défectueux VD114 ou VD180 par le variant SK. II est aussi possible qu'une infection double d'un hôte par les variants VD114 et VD180 puisse donner le profil SK, par le jeu d'une complémentation sérologique complète. Cependant, cette possibilité ne met nullement en doute l'existence du variant sérologique SK qui possède des propriétés totalement différentes de celles de VD114 ou de VD180 (incapacité d'infecter les hôtes spécifiques de VD114, induction de symptômes différents de ceux induits par VD114 et VD180).

Le maïs, malgré la mise en évidence d'infections doubles, a toujours exhibé le profil de réaction SK. Ce résultat ne peut s'expliquer que par un masquage sérologique systématique des variants sérologiques VD114 et VD180 par SK. La reconstitution in vitro du profil de réaction hybride montre que le masquage sérologique résulte $d^{\prime}$ une plus grande accumulation des particules virales du variant qui masque. Ce masquage peut être considéré comme le résultat d'une adaptation particulière du SK au maïs et la principale cause de la difficulté de distinguer sérologiquement

Tableau 2. Réaction de cinq hôtes du MSV envers trois variants sérologiques du MSV

\begin{tabular}{|c|c|c|c|c|c|c|}
\hline \multirow[b]{2}{*}{ Hôtes } & \multicolumn{3}{|c|}{ Symptômes } & \multicolumn{3}{|c|}{ Test ELISA } \\
\hline & $\mathrm{SK}^{\mathrm{a}}$ & VD114 & VD180 & $\mathrm{SK}^{\mathrm{a}}$ & VD114 & VD180 \\
\hline Brachiaria deflexa & $0^{b}$ & 15 & 0 & $-c$ & + & - \\
\hline $\begin{array}{l}\text { Oryza sativa } \\
\mathrm{cv} \text {. sindano }\end{array}$ & 0 & 20 & 0 & - & + & - \\
\hline $\begin{array}{l}\text { Pennisetum glaucum } \\
\text { cv. kotra }\end{array}$ & 0 & 12 & 0 & - & + & - \\
\hline Rottboellia exaltata & 0 & 8 & 0 & - & + & - \\
\hline Setaria pallide-fusca & 0 & 16 & 0 & - & + & - \\
\hline
\end{tabular}

a Variant SK pouvant avoir des réactions de type $\mathrm{SK}_{\mathrm{F}}$ ou $\mathrm{SK}_{\mathrm{f}}$.

b Nombre de plantes infectées pour un total de 20 plantes testées.

c + : réaction positive; - : réaction négative. 


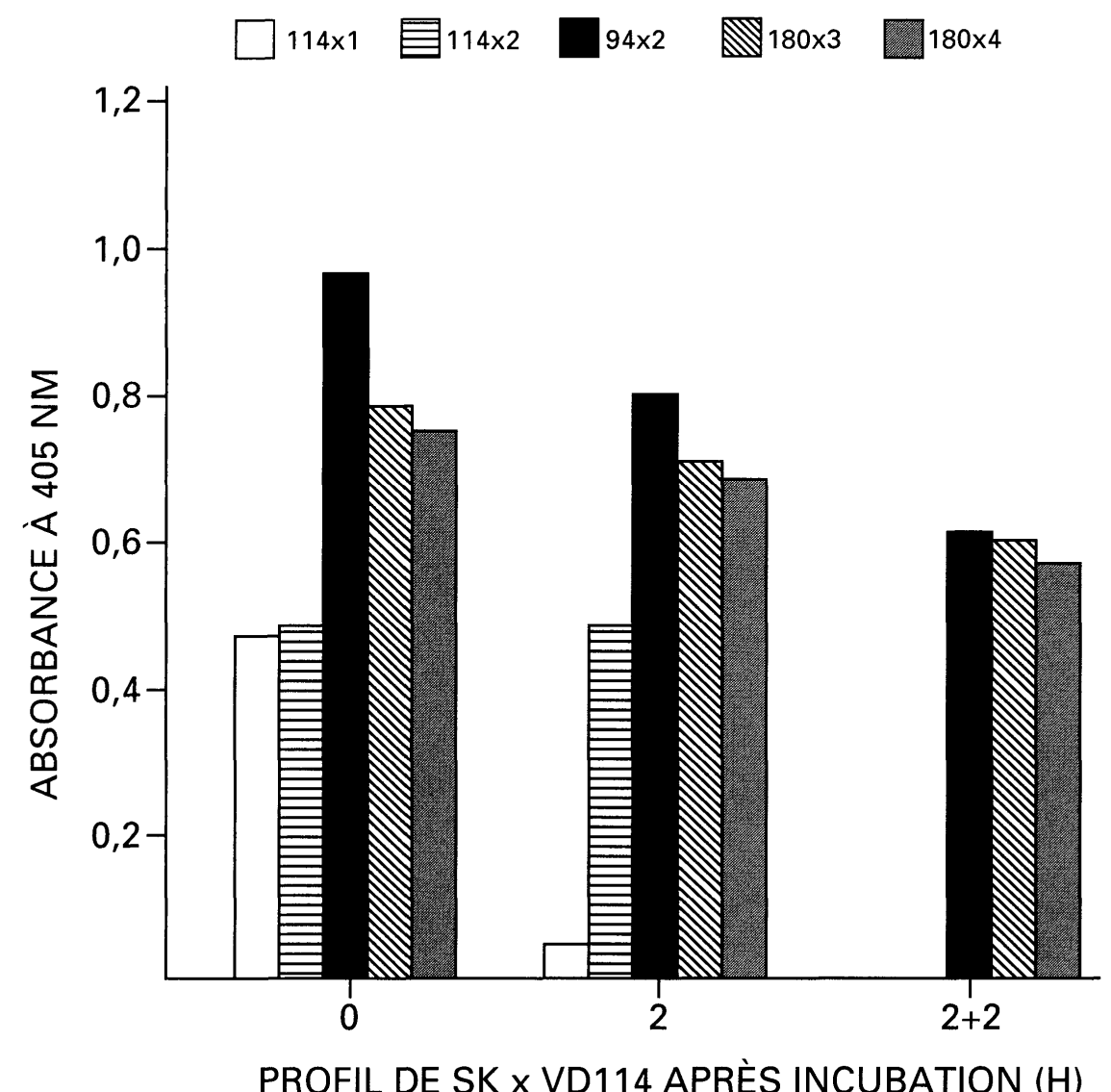

Figure 3. Épuisement sérologique des particules virales du variant SK dans un extrait de feuille infectée exhibant le profil de réaction hybride SK $x$ VD114. $0=$ profil hybride initial; $2=$ profil après $2 \mathrm{~h}$ d'incubation dans les alvéoles; $2+2=$ profil après $2 \mathrm{~h}$ supplémentaires d'incubation.

les isolats maïs du MSV. De même, les symptômes prononcés de $\mathrm{SK}_{\mathrm{F}}$ masquent systématiquement ceux de VD114, VD180 et SK $_{f}$. II n'y a donc aucune protection croisée contre $\mathrm{SK}_{\mathrm{F}}$. II s'agit là d'un désavantage pour le maïs. L'infection double et le masquage sérologique et symptomatique sont en accord avec les résultats de Dekker et al. (1988) et de Pinner et al. (1988).

Nos résultats montrent que deux variants du MSV, ayant des épitopes et une gamme d'hôtes différents, VD114 et VD180, peuvent induire des symptômes identiques chez le maïs et que deux sous-variants du MSV, ayant les mê- mes épitopes reconnus par les anticorps monoclonaux, $\mathrm{SK}_{\mathrm{F}}$ et $\mathrm{SK}_{\mathrm{f}}$, peuvent induire des symptômes totalement différents apparaissant, par contre, après le même délai. Ces résultats peuvent s'interpréter à la lumière des travaux de Boulton et al. (1991a, 1991b). Dans ces travaux, la gamme d'hôtes du MSV et les symptômes induits étaient déterminés par des domaines spécifiques du génome viral. Un changement d'un seul nucléotide suffisait pour affecter la gamme d'hôtes, les symptômes et le délai d'apparition de ces derniers. Un tel changement peut ne pas affecter la protéine de capside, responsable des propriétés antigéniques du virus. 
Les caractéristiques sérologiques et pathogéniques du sous-variant $\mathrm{SK}_{\mathrm{F}}$ le rapprochent de plusieurs isolats du MSV caractérisés par différents auteurs (Bock et al.1974; Dekker et al. 1988; McClean 1947; Peterschmitt et al.1991; Pinner et Markham 1990; Rose 1978). Celles du $\mathrm{SK}_{\mathrm{f}}$ le rapprochent de I'isolat SP atténué décrit par Peterschmitt et al. (1991). Quant au variant VD114, ses caractéristiques le rapprochent des isolats $M(N) M$ M1 (Pinner et al. 1988) et MSV-Nm (Boulton et al. 1991b). Par contre le VD180 ne ressemble à aucun isolat décrit.

Un seul des trois sérotypes rapportés par Peterschmitt et al. (1991) est rencontré au Burkina Faso. II s'agit du sérotype SP dont le variant pathogénique SP prononcé avait été préalablement détecté au Burkina Faso par ces auteurs, chez la canne à sucre.

En condition naturelle, le maïs est infecté par des variants sérologiques et symptomatiques très différents. Cependant, la puissante capacité de masquage du variant $\mathrm{SK}_{\mathrm{F}}$ fait que tous les échantillons de maïs infectés au champ apparaissent uniformes. Par contre, nos études sur une gamme étendue d'hôtes du MSV, nous ont permis d'identifier cinq hôtes, spécifiques au variant VD114, qui sont résistants aux autres variants. Ces résultats, associés à ceux obtenus par des études sérologiques, constituent le premier succès dans la distinction d'isolats maïs du MSV par la sérologie et par la symptomatologie en conditions d'infection naturelle.

\section{REMERCIEMENTS}

Nous remercions les docteurs M.H.V. Van Regenmortel, B. Huguenin et P. Sérémé pour la lecture critique du manuscrit, et $M$. Peterschmitt pour la fourniture des anticorps monoclonaux. Ce travail a été financé par le Ministère français de la Coopération et du Développement.

\section{RÉFÉRENCES}

Bock, K.R., E.J. Guthrie et R.D. Woods. 1974. Purification of maize streak virus and its relationship to viruses associated with streak diseases of sugar cane and Panicum maximum. Ann. Appl. Biol. 77: 289296.
Boulton, M.I., D.I. King, J. Donson et J.W. Davies. 1991a. Point substitutions in a promoter-like region and the $\mathrm{V} 1$ gene affect the host range and symptoms of maize streak virus. Virology 183: 114-121.

Boulton, M.I., D.I. King, P.G. Markham, M.S. Pinner et J.W. Davies. 1991b. Host range and symptoms are determined by specific domains of the maize streak virus genome. Virology 181: 312-318.

Clark, M.F. et A.N. Adams. 1977. Characteristics of the microplate method of ELISA for detection of plant viruses. J. Gen. Virol. 34: 475-483.

Dekker, E.L., M.S. Pinner, P.G. Markham et M.H.V. Van Regenmortel. 1988. Characterization of maize streak virus isolates from different plant species by polyclonal and monoclonal antibodies. J. Gen. Virol. 69: 983-990.

Dollet, M., G.P. Accoto, V. Lisa, J. Menissier et G. Boccardo. 1986. A geminivirus, serologically related to maize streak virus, from Digitaria sanguinalis from Vanuatu. J. Gen. Virol. 67: 933-937.

Francki, R.I.B., R.G. Milne et T. Hatta. 1985. Geminivirus group. Pages 33-46 in R.I.B. Francki, R.G. Milne et T. Hatta (réd.), Atlas of plant viruses. CRC Press, Boca Raton, Floride.

Fuller, C. 1901. Mealie variegation. First report of government entomologist 18991900, Natal: 17-19.

Harrison, B.D. 1985. Advances in geminivirus research. Annu. Rev. Phytopathol. 23: 55-82.

Konaté, G. et O. Traoré. 1992. Les hôtes réservoirs du virus de la striure du maïs (MSV) en zone soudano-sahélienne: identification et distribution spatio-temporelle. Phytoprotection 73: 111-117.

McClean, A. 1947. Some forms of streak virus occurring in maize, sugar cane and wild grasses. Bull. Sci., Dep. Agric. Tech. Serv. Univ. S. Afr. 265: 12-39.

Mullineaux, P.M., J. Donson, B.A.M. MorrisKrsinich, M.I. Boulton et J.W. Davies. 1984. The nucleotide sequence of maize streak virus DNA. EMBO J. 3: 306:3-3068.

Peterschmitt, M., B. Reynaud, G. Sommermeyer et P. Baudin. 1991. Characterization of maize streak virus isolates using monoclonal and polyclonal antibodies and by transmission to a few hosts. Plant Dis. 75: 27-32.

Pinner, M.S. et P.G. Markham. 1990. Serotyping and strain identification of maize streak virus isolates. J. Gen. Virol. 71: 1635-1640.

Pinner, M.S., P.G. Markham, R.H. Markham et E.L. Dekker. 1988. Characterization of maize streak virus: Description of strains; symptoms. Plant Pathol. 37: 74-87. 
Ricaud, C. et S. Félix. 1978. Strains of streak virus infecting sugar cane. Proc. Inst. Soc. Sugar Cane Technol. 16: 449-457.

Roberts, I.M., D.J. Robinson et B.D. Harrison. 1984. Serological relationships and genome homologies among geminiviruses. J. Gen. Virol. 65: 1723-1730.

Rose, D.J.W. 1978. Epidemiology of maize streak disease. Annu. Rev. Entomol. 23: 259-282.

Seth, M.L., S.P. Raychaudhuri et D.V. Singh. 1972. Bajra (Pearl millet) Streak, a leafhopper-borne cereal virus in India. Plant Dis. Rep. 56: 424-428.

Stanley, J. 1985. The molecular biology of geminiviruses. Adv. Virus Res. 30: 139177.
Storey, H.H. et A. McClean. 1930. The transmission of streak disease between maize, sugar cane and wild grasses. Ann. Appl. Biol. 17: 691-719.

Van Regenmortel, M.H.V. et J. Burckard. 1980. Detection of a wide spectrum of tobacco mosaic virus strains by indirect enzyme-linked immunosorbent assay (ELISA). Virology 106: 327-334.

Voller, A., A. Barlett, D.E. Bidwell, M.F. Clark et A.N. Adams. 1976. The detection of viruses by enzyme-linked immunosorbent assay (ELISA). J. Gen. Virol. 33: 165-167.

Webb, D.M. 1987. Species recognition of Cicadulina leafhoppers (Hemiptera: Cicadellidae), vectors of pathogens of Gramineae. Bull. Entomol. Res. 77: 683-712. 